\title{
Digitalisierung - Potential und Grenzen der Analogie zum Analogen
}

\author{
Herbert Zech ${ }^{1}$
}

Vergleiche zwischen analoger und digitaler Welt gehören zum Standardrepertoire der Beschäftigung mit der Digitalisierung. Sie liefern einen ersten Anhaltspunkt für das Verständnis der Funktionsweise digitaler Lösungen und ermöglichen die Beurteilung ihrer Auswirkungen. Verschiedene rechtliche Diskussionen zu Phänomenen der Digitalisierung haben aber gezeigt, dass eine vorschnelle Gleichsetzung mit Bekanntem aus der nichtdigitalen Welt zu fehlerhaften Beurteilungen führen kann. Pauschal lässt sich nicht beantworten, ob es letztlich mehr schadet als nützt, wenn wir in Vergleichen zwischen analogen und digitalen Sachverhalten denken. Der folgende Beitrag soll die Probleme solcher Vergleiche bei der Rechtsanwendung und -fortbildung bewusst machen.

\section{A. Digitales und Analoges: Was bedeutet Digitalisierung?}

\section{Digitalisierung als Einführung von Informationstechnologie}

Digitale Systeme leisten Informationsverarbeitung. Während eine solche auch von Menschen geleistet werden kann (der Begriff Computer bezeichnete ursprünglich Menschen, die rechnen ${ }^{2}$ ), ist mit der Informationstechnologie die Möglichkeit der maschinellen Informationsverarbeitung entstanden. ${ }^{3}$ Der Begriff digital, der sich ursprünglich vom Rechnen mit Fingern (lat. digitus, der Finger) herleitet, wird häufig synonym mit informa-

1 Prof. Dr. jur. Dipl.-Biol., Lehrstuhl für Bürgerliches Recht, Technik- und IT-Recht, Humboldt-Universität zu Berlin, Direktor am Weizenbaum-Institut für die vernetzte Gesellschaft. Für zahlreiche Anmerkungen und Hinweise danke ich Lisa Lueg, LL.M. (Cambridge) und Paul Dürr.

2 Zumeist Frauen, die in Forschungsinstituten Rechenaufgaben übernommen haben, dazu z.B. Smith, CLC Vol. 15, Iss. 1 (2013), abrufbar unter https://doi.org/10.7 771/1481-4374.1972<1.5.2020>.

3 Bermudez, Cognitive Science, 2. Aufl. 2014, S. 2: „The most fundamental driving assumption of cognitive science is that minds are information processors." 
tionstechnisch verwendet. Innerhalb der Informationstechnik steht digital für die Informationsverarbeitung in diskreten Schritten, insbesondere im binären Zahlensystem, während analog eine Informationsverarbeitung durch kontinuierliche Zustandsänderung bedeutet. Da heute jedoch die digitale Informationstechnologie dominiert, ist es durchaus gerechtfertigt, den Begriff digital als pars pro toto für informationstechnisch zu verwenden. Analog soll daher hier das Gegenteil von digital bedeuten.

Die Digitalisierung kann als die Einführung digitaler Systeme und damit technischer Informationsverarbeitung in bestimmten Lebensbereichen beschrieben werden. Der Prozess der Digitalisierung erstreckt sich heute auf nahezu alle Lebensbereiche. Dies wurde durch Fortschritte (neutral formuliert Entwicklungen) in der Informationstechnologie ermöglicht, die digitale Systeme immer leistungsfähiger gemacht haben. Zu den neuesten Fortschritten gehören der Einsatz digitaler Systeme zur unmittelbaren Hardwaresteuerung (Robotik), die Lernfähigkeit digitaler Systeme und deren zunehmende Vernetzung. ${ }^{4}$

\section{Von der Informationsübermittlung zur künstlichen Intelligenz}

Historisch hat sich die Digitalisierung, grob zusammengefasst, vom einfachen Rechnen über die Informationsübermittlung (insbesondere durch das Internet) hin zu den dargestellten neueren Entwicklungsfeldern Robotik, Lernfähigkeit und Internet of Things entwickelt. Es lassen sich zahlreiche Beispiele für Aufgaben nennen, die im Zuge dieser Entwicklung digitalisiert wurden (teilweise sind natürlich auch neuartige Aufgaben entstanden): Durch das Phänomen des Internets wurde die Informationsübermittlung bzw. Nachrichtenübermittlung oder ganz allgemein die Kommunikation zwischen Menschen digitalisiert (Internet, Email, digitale Medien). Ein neueres Phänomen ist die Erfassung und Analyse von Daten, die beim Betrieb digitalisierter Geräte anfallen (Big Data). Lernfähige digitale Systeme wurden bereits erwähnt. Sie sind ein wesentlicher Schritt hin zu einer Form künstlicher Intelligenz, die dieser Bezeichnung gerecht werden kann.

4 Zech, Weizenbaum Series \#2, 2020, Risiken digitaler Systeme: Robotik, Lernfähigkeit und Vernetzung als aktuelle Herausforderungen für das Recht, abrufbar unter https://www.weizenbaum-institut.de/media/News/Weizenbaum_Series/Weizenbau m_Series_2_Zech_070220.pdf $<1.5 .2020>$. 
Mit der wachsenden Leistungsfähigkeit vergrößert sich auch das Funktionsspektrum digitaler Systeme. Immer mehr Aufgaben, die früher nur von Menschen erledigt werden konnten, lassen sich inzwischen digital bewältigen. Ein Vergleich von maschinell erfüllbaren mit von Menschen erfüllbaren Aufgaben im Bereich der Informationsverarbeitung liegt auch dem Begriff der künstlichen Intelligenz zugrunde. Künstliche Intelligenz bezeichnet den Versuch, Leistungen natürlicher Intelligenz durch digitale Systeme erbringen zu lassen. Dazu gehören etwa Wahrnehmung und Mustererkennung, Wissensrepräsentation, Problemlösung, Schlussfolgern, Entscheiden, Planung bis hin zu emotionaler Intelligenz und Kreativität.

\section{Informationstechnologie als transformative Technologie}

Die Verbreitung digitaler Systeme in allen Aspekten des Lebens bedingt ihre stetig wachsende Relevanz für soziale Prozesse. ${ }^{5}$ Informationstechnologie hat dabei transformatives Potential: Sie ist in ihren Auswirkungen ambivalent und schwer prognostizierbar, kann grundlegende gesellschaftliche Praktiken und Wertevorstellungen nicht nur beeinflussen, sondern sogar einem tiefgreifenden Wandel unterwerfen. Sie ist damit eine transformative Technologie ${ }^{6}$ bzw. defining technology ${ }^{7}$ unserer Zeit.

5 Brynjolfsson/McAfee, The Second Machine Age: Work, Progress, and Prosperity in a Time of Brilliant Technologies, 2014, S. 90: "the exponential, digital, and recombinant powers of the second machine age have made it possible for humanity to create two of the most important one-time events in our history: the emergence of real, useful artificial intelligence (AI) and the connection of most of the people on the planet via a common digital network".

6 Zum Begriff der transformativen Technologie Nuffield Council on Bioethics, Genome editing: an ethical review, 2016, S. 12, 26; Fateh-Moghadam, medstra 2017, 146 (148); ders., ZStW 2019, 863 (865 ff.). Aktuell werden also Genomeditierung und künstliche Intelligenz als die transformativen Technologien bzw. defining technologies unserer Zeit diskutiert. Eine zuverlässige Beurteilung ist natürlich immer erst retrospektiv möglich.

7 Bolter, Turing's Man: Western Culture in the Computer Age, 1984, S. 8 f. Bereits hier findet sich ein Ausblick auf die zunehmende gesellschaftliche Bedeutung der Informationstechnologie (S.9): "For us today, the computer constantly threatens to break out of the tiny corner of human affairs (scientific measurement and business accounting) that it was built to occupy, to contribute instead to a general redefinition of certain basic relationships: the relationship of science to technology, of knowledge to technical power, and, in the broadest sense, of mankind to the world of nature." 


\section{B. Vergleiche digitaler und analoger Lebenssachverhalte als Grundlage für Analogien (insbesondere rechtlicher Analogieschlüsse)}

\section{Vergleich als Grundlage von (rechtlichen) Analogieschlüssen}

Sämtlichen Analogien liegt ein Vergleich zwischen zwei Objekten zugrunde. Ergibt der Vergleich, dass zwei Objekte ähnlich sind bzw. gleiche Merkmale aufweisen, können im Wege des Analogieschlusses weitere Merkmale des einen Objekts auf das andere übertragen werden. Methodisch handelt es sich bei dem Analogieschluss damit nicht um eine zwingende logische Schlussfolgerung, sondern um eine Argumentationsfigur, deren Überzeugungskraft stark von der Plausibilität des zugrundeliegenden Vergleichs abhängig ist. ${ }^{8}$

Bei der rechtlichen Analogie werden zwei Sachverhalte miteinander verglichen, nämlich zum einen ein konkreter oder typisierter Sachverhalt, der $\mathrm{zu}$ beurteilen ist, und zum anderen ein typisierter Sachverhalt, der einer Norm, die analog angewendet werden soll, als Tatbestand zugrunde liegt. Der rechtliche Analogieschluss hat zwei Voraussetzungen: Zum einen das Vorliegen einer planwidrigen Regelungslücke und zum anderen die wertungsmäßige Vergleichbarkeit der Interessenlage. Um letztere festzustellen, werden der Tatbestand (und damit der typische Sachverhalt, der einen Tatbestand erfüllt, Normfall) als normativer Referenzpunkt und der tatsächliche Lebenssachverhalt (bzw. der zu beurteilende Sachverhalt) einander im Lichte der analog anzuwenden Norm gegenübergestellt.

\section{Digitalisierung kann zu planwidrigen Regelungslücken fübren}

Die Digitalisierung eines Lebensbereichs kann dazu führen, dass bestehende Normen nicht mehr eindeutig auf die dort rechtlich zu beurteilenden Lebenssachverhalte passen. ${ }^{9}$ So lässt sich beispielsweise bei elektronischer Kommunikation das Briefgeheimnis, wenn man strikt auf den Wortlaut des Art. 10 Abs. 1 GG abstellt, nicht mehr anwenden. Werden Medien per

8 Menne, Einführung in die Logik, 5. Aufl., 1993, S. 19: „Folgerichtigkeit setzt Eindeutigkeit voraus. Analogie und Metapher aber sind Mehrdeutigkeiten." Vgl. zur Auseinandersetzung mit den logischen Hintergründen des rechtlichen Analogieschlusses Heller, Logik und Axiologie der analogen Rechtsanwendung, 1961, S. 7 ff., sowie Klug, Juristische Logik, 4. Aufl. 1982, S. 115 ff.

9 Hoffmann-Riem, Innovation und Recht - Recht und Innovation, 2016, S. 113 ff.: Veränderung des „Realbereichs“ von Normen. 
Streaming konsumiert, fehlt es an physischen Ton- oder Bildträgern, deren Vermarktung bestimmten urheberrechtlichen Regeln unterliegt. Hinzu kommt, dass die Digitalisierung auch zu neuartigen Verhaltensweisen führen kann, wie etwa der Behandlung von Daten als handelsfähiges Gut, für die es keine passenden rechtlichen Regelungen gibt. Da die Digitalisierung gerade ein Phänomen gesellschaftlicher Veränderung darstellt, erweisen sich planwidrige Regelungslücken als typische Folge der Digitalisierung auf dem Gebiet der Rechtsanwendung. Rechtsmethodisch kann ein solches normatives Vakuum durch das Instrument des Analogieschlusses geschlossen werden.

\section{Vergleich digitaler und analoger Sachverhalte}

Digitalisiert werden können alle Aspekte des menschlichen Lebens, sofern nur digitale Systeme als Hilfsmittel eingesetzt werden können. Angelehnt an die rechtliche Terminologie kann man von digitalen Sachverhalten sprechen.

Als Bezugspunkt für den Vergleich bietet sich der gleiche Lebenssachverhalt ohne Einsatz digitaler Hilfsmittel an. Man kann von analogen Sachverhalten sprechen. Digitale und analoge Lebenssachverhalte können diachron oder synchron verglichen werden. Häufig handelt es sich (da Digitalisierung einen Entwicklungsprozess beschreibt) um einen diachronen Vergleich, es wird also ein früherer analoger mit einem aktuellen bzw. neuartigen digitalen Sachverhalt verglichen. Allerdings ist dies nicht zwingend und es können durchaus auch einander entsprechende analoge und digitale Sachverhalte gleichzeitig auftreten (ein Beispiel für Musikliebhaber wäre das Fortleben von Vinylplatten neben CDs und Streaming).

Möchte man nun eine vorhandene Norm, die für einen analogen Sachverhalt geschaffen wurde, auf einen digitalen Sachverhalt anwenden, so ergibt sich ein einfaches Muster: Tatbestandlicher und zu beurteilender Sachverhalt entsprechen analogem und digitalem Sachverhalt. Es wird also ein digitaler (nicht ausdrücklich normierter) mit einem analogen (normierten) Sachverhalt verglichen. 


\section{Ergebnis des Analogieschlusses: Herleitung deskriptiver und normativer Aussagen}

\section{Umfang des Ergebnisses: übertragene Eigenschaften}

Analogien können danach unterschieden werden, zu welcher Art von Schlüssen sie führen. Auch kann eine Analogie hinsichtlich der Folgen bzw. der Zahl der übertragenen Merkmale mehr oder weniger umfassend sein. Entsprechend kann eine Analogie auch hinsichtlich der Voraussetzungen, also der Zahl der verglichenen Merkmale, mehr oder weniger umfangreich sein (dazu unter D.). Die Überzeugungskraft eines Analogieschlusses hängt von mehreren Parametern ab: Einerseits von der Anzahl der verglichenen Merkmale, andererseits von deren jeweiliger Relevanz.

\section{Herleitung deskriptiver und normativer Aussagen}

Wichtig ist, beim Analogieschluss zwischen der Herleitung deskriptiver und normativer Aussagen zu unterscheiden. Bei rechtlichen Analogieschlüssen wird stets eine normative Aussage hergeleitet, während Aussagen über bestimmte Eigenschaften von Gegenständen der uns umgebenden Welt deskriptiven Charakter haben.

Wenn eine normative Aussage gewonnen werden soll, muss die Analogie sich eines normativen Referenzpunktes bedienen. Normative Schlüsse lassen sich nicht aus deskriptiven Aussagen ableiten. Dies ist allerdings bei rechtlichen Analogien selbstverständlich, da es bei ihnen um die analoge Anwendung von Normen geht. Festzuhalten bleibt aber, dass der Vergleich sowohl deskriptive als auch normative Komponenten betrifft. Deskriptive Komponenten ergeben sich aus dem Vergleich der Sachverhalte normative aus dem Vergleich der rechtlich relevanten Interessenlagen. Aus der Ähnlichkeit analoger und digitaler Sachverhalte, einer deskriptiven Beurteilung, auf die Anwendung einer Norm zu schließen, ist dabei wie jede Subsumtion zulässig. Allerdings muss die Ähnlichkeit auch mit einer Vergleichbarkeit der Interessenlage einhergehen. Nur dann ist die rechtliche Analogie zulässig.

Damit stellt sich die Frage, welche Interessen bzw. Wertungsgesichtspunkte beim Vergleich der Interessenlage berücksichtigt werden müssen, wann also der Interessenvergleich vollständig ist. Welche Interessen dabei Gewicht haben und welches Gewicht ihnen zukommt, entscheidet das Recht. 


\section{Veranschaulichung der technischen Funktion digitaler Systeme}

Bevor weiter das Potential von Rechtsanalogien untersucht wird, soll auf Vergleiche zwischen analoger und digitaler Welt mit rein deskriptivem Aussagegehalt eingegangen werden. Denn allein die Tatsache, dass eine Analogie sich auf dem Feld deskriptiver Aussagen bewegt, bedeutet nicht, dass sie für juristische Diskussionen keine Relevanz besitzt. Im Gegenteil: Für die Rechtsanwendung ist stets auch ein solides Verständnis des jeweiligen Lebenssachverhalts erforderlich. Bei der Veranschaulichung digitaler Systeme und ihrer technischen Funktionsweise können deskriptive Vergleiche sehr nützlich sein. Damit sind wir in einem ersten Bereich, in dem wir das Potential von Analogien zum Analogen bewerten können und in dem die Bewertung positiv ausfällt.

Ein Beispiel aus der juristischen Literatur ist der Vergleich von Information und Wasser. ${ }^{10}$ So kann etwa Information objekthaft wie Wasser gespeichert oder über Kanäle übertragen werden. Interessant ist, dass es ein ähnliches Bild in der Physik für die Stromübertragung gibt, bei der die Stromstärke dem Wasserfluss entspricht und die Stromspannung dem Wasserdruck. Damit wird deutlich, dass das Denken in Vergleichen auch den Naturwissenschaften nicht fremd ist, worauf kurz eingegangen werden soll.

\section{Modelldenken in den Naturwissenschaften}

Auch in den Naturwissenschaften werden Analogien verwendet, um sich neuartigen - und auch altbekannten - Problemen anzunähern. Hier hat die Verwendung von Modellen eine zentrale Bedeutung. ${ }^{11}$ Auch wenn sich ein Modell zu einem späteren Zeitpunkt als unvollständig herausstellen mag, kann es doch neue Erkenntnisse ermöglichen. ${ }^{12}$

10 Dreier, Von Gütern, Kanälen und Speichern - Metaphern des Informationsrechts, Festvortrag, gehalten am 1. Dezember 2001, anläßlich der Jahresfeier der Universität Fridericiana zu Karlsruhe, https://www.zar.kit.edu/DATA/ recht/veranst/drei er2001/Festrede.pdf <1.5.2020>: "Informationen in bildhafter Analogie zu Gütern, Kanälen und Speichern vorstellen“; von Lewinski, Datenflut und Recht - Informationsrecht als Deich, Kanal, Wasserhahn oder Rettungsring?, 2013.

11 Dazu Rosenberg, Philosophy of Science, 3. Aufl. 2012, S. $162 \mathrm{ff}$.

12 Pointiert etwa zur Erklärung der Aggregatzustände mit der Teilchenvorstellung bzw. der kinetischen Theorie der Wärmelehre Christen, Struktur Stoff Reaktion, Allgemeine und Anorganische Chemie, 1974, S. 11: „Naturwissenschaftli- 
Ein gutes Beispiel ist die kalorische Theorie in der Wärmelehre, in der im 18. Jahrhundert (und noch bis ins 19. Jahrhundert hinein) Wärme als ein Stoff behandelt wurde. Obwohl sich diese Theorie als falsch herausstellte und durch die kinetische Theorie ersetzt wurde, erlaubte sie dennoch bereits wichtige Erkenntnisse wie die mathematische Beschreibung von Wärmekraftmaschinen durch Sadi Carnot. Ein anderes häufig verwendetes Beispiel ist das geozentrische Planetenmodell, das durch das heliozentrische ersetzt wurde. Gegenüber dem einfachen Modell von Kopernikus war das zeitgenössische geozentrische Modell (etwa in der Ausarbeitung von Tycho Brahe) überlegen, d.h. es sagte die Bewegung der Planeten am Sternenhimmel genauer voraus. Erst mit der Weiterentwicklung durch Kepler, der die kreisförmigen Planetenbahnen durch elliptische ersetzte, wurde das heliozentrische Modell leistungsfähiger.

\section{Vorläufigkeit der gewonnenen Aussagen}

Aus dem Exkurs in die Naturwissenschaften wird deutlich, dass viele Vergleiche und die aus ihnen gezogenen Schlüsse vorläufigen Charakter haben. Folge ist, dass das Modell jederzeit auch wieder aufgegeben und durch ein besseres ersetzt oder ergänzt werden kann. Dies ist gerade in den Naturwissenschaften allgemein akzeptiert und gehört zu den Grundfragen der Wissenschaftsphilosophie. Unvollständigkeit ist Teil des Programms.

$\mathrm{Da}$ aber in den Naturwissenschaften keine normativen Aussagen hergeleitet werden, droht dadurch kein Gerechtigkeitsproblem, wobei die Unvollständigkeit aber durchaus z.B. ein Problem des technischen Funktionierens bedingen kann (wenn das Modell einer Technologie zugrunde liegt). Anders verhält es sich bei rechtlichen Analogieschlüssen. Leitet man eine normative Aussage her, führt ein fehlerhafter Schluss zu einer fehlerhaften Rechtsanwendung und kann zu einem Gerechtigkeitsproblem führen. Auch aus dem Gesichtspunkt der Rechtssicherheit bestehen Bedenken gegen eine bewusste „Inkaufnahme“ fehlerhafter rechtlicher Analogieschlüsse: Eine kontinuierliche Verfestigung zu Richterrecht erscheint da-

ches 'Verstehen' oder 'Erklären' bedeuten also letztlich nichts anderes, als daß man gewisse Erscheinungen oder Beobachtungen auf etwas bereits Bekanntes zurückführt oder durch ein Modell, das gewissen Erfahrungen entspricht, beschreibt. [...] Man muß sich dabei allerdings hüten, das Modell mit der Wirklichkeit gleichzusetzen; jedes Modell ist zwangsläufig in mehr oder minderem Maß vereinfacht und muß ständig verbessert, d.h. verfeinert werden, um weiteren Beobachtungen gerecht zu werden, d.h. um auch diese zu 'erklären'.“ 
mit nicht vereinbar. Zwar kann auch Gesetzes- wie Richterrecht geändert werden. Dennoch ist ein vorläufiger Charakter normativer Aussagen im Sinne einer Korrigierbarkeit im konkreten Fall mit dem autoritativen Charakter von Rechtsnormen nicht zu vereinbaren (sieht man einmal von gesetzlich geregelten Fällen wie der zulässigen Rücknahme eines rechtswidrigen Verwaltungsakts ab). Zwar kann abstrakt-generelles Recht geändert werden (wenn auch unter Beachtung des Rückwirkungsverbots), nicht aber individuell-konkrete Rechtsanwendung (Gedanke der Rechtskraft). Rechtliche Analogien sind keine Experimentierfelder, in denen die Lösungsfindung im trial and error-Verfahren erfolgen kann. Vielmehr ist bei der Annahme einer Analogie ein hohes Maß an normativer Begründungstiefe erforderlich.

\section{Unvollständige und vollständige Vergleiche}

\section{Umfang des Vergleichs: Verglichene Merkmale}

Analogien können auch danach unterschieden werden, wie umfangreich der ihnen zugrunde liegende Vergleich ist. Dies richtet sich nach der Zahl der verglichenen Merkmale und deren Relevanz. Die Zahl der verglichenen Merkmale ist dabei nicht absolut zu sehen, sondern im Verhältnis der möglichen Vergleichsmerkmale.

Die Rechtsanalogie, die sich auf mehrere Normen bezieht, ${ }^{13}$ scheint eine Analogie zu sein, die auf einem umfangreichen Vergleich beruht. Dies ist jedoch nicht zwingend der Fall, da sich dabei häufig erst aus der Zusammenschau mehrerer Normen hinreichende Anhaltspunkte für eine Analogie ergeben.

\section{Vollständigkeit als Voraussetzung des rechtlichen Analogieschlusses}

Ein vollständiger Vergleich würde sämtliche Merkmale außer den zu übertragenden erfassen. Freilich wird dies kaum je positiv feststellbar sein, da sich regelmäßig auch neue vergleichbare Merkmale finden lassen.

13 Ein Beispiel für eine solche Rechts- bzw. Gesamtanalogie wäre die Gefährdungshaftung für Technik, die man im Wege einer Gesamtanalogie zu den bestehenden Technikhaftungsregelungen bejahen kann; s. Kötz/Wagner, Deliktsrecht, 13. Aufl. 2017, S. 208 f.; Zech, in: Jahrbuch SGHVR 2016, S. 17, 32. 
Insbesondere bei rechtlichen Analogien ist aber die Vollständigkeit des Vergleichs der Interessenlage Voraussetzung für den Analogieschluss. Vollständigkeit des Vergleichs bedeutet hier Berücksichtigung aller aus normativer bzw. rechtlicher Sicht relevanter Interessen.

Die Vollständigkeit des Vergleichs der Interessenlage ist daher immer nur eine relative: Die Vollständigkeit eines Vergleichs ist mit Blick auf einen bestimmten Gesetzeszweck zu beurteilen. Hier kommen Nachbardisziplinen wie die ökonomische Analyse oder die Digitalisierungsforschung zum Tragen, im Kern bleibt es aber eine rechtliche Interessenanalyse, die Grundlage des Vergleichs ist.

\section{III. Ökonomische Analyse}

Häufig ändert sich durch die Digitalisierung gerade die volkswirtschaftliche Bedeutung bestimmter Sachverhalte. Weil Digitalisierung erleichterte Übertragung, Speicherung und Verarbeitung von Information bedeutet, hat sie auch Auswirkung auf damit verbundene wirtschaftliche Prozesse (heutzutage praktisch alle). Die Digitalisierung bewirkt nicht nur ungeheure Effizienzgewinne, sondern schafft häufig auch neue Geschäftsmodelle, die alte ablösen, womit auch wieder Vergleichspaare für Analogien entstehen.

Eine rechtliche Analyse kann daher die wirtschaftliche Gemengelage nicht ausblenden. Deshalb gewinnt die ökonomische Analyse des Rechts gerade auch im Hinblick auf die normativen Auswirkungen der Digitalisierung eine hohe Bedeutung. Bei dem Interessenvergleich im Rahmen eines Analogieschlusses kann sie wertvolle Anhaltspunkte liefern.

Selbstverständlich gibt es auch andere wichtige Aspekte, die bei einem Vergleich herangezogen werden können oder müssen (je nach dem konkreten Vergleichspaar). Dazu gehören unter anderem grundrechtliche Auswirkungen und soziale Aspekte wie etwa Auswirkungen auf Demokratie und gesellschaftliche Teilhabe.

\section{Sonstige relevante Interessen}

Die Ökonomik beschäftigt sich mit der Verteilung knapper Güter zur Befriedigung menschlicher Bedürfnisse. Das Recht schützt allerdings nicht nur individuelle bzw. aggregierte Interessen an einer bestimmten Verteilung knapper materieller Güter, sondern auch ideelle Interessen (siehe et- 
wa den Persönlichkeitsschutz). Auch andere, für die Erfassung der Interessenlage im Privatrecht relevante Nachbardisziplinen kommen neben der ökonomischen Analyse in Betracht, z.B. die Philosophie.

Die Eingrenzung erfolgt anhand normativer Überlegungen. Es sind also alle rechtlich geschützten Interessen bzw. Werte zu ermitteln, die bei einer bestimmten Fallgestaltung tangiert sind. Diese sind beim Vergleich der Interessenlage vollständig zu berücksichtigen.

\section{E. Der Gedanke der funktionalen Ersetzung}

\section{Funktionale Ersetzung als typischer Aspekt der Digitalisierung}

Ein wichtiges Beispiel für Analogien, die zumindest Gefahr laufen, auf einem unvollständigen Vergleich zu beruhen, sind solche, die an den Gedanken der funktionalen Ersetzung anknüpfen. Dabei handelt es sich um einen typischen Aspekt der Digitalisierung als Phänomen technischer Entwicklung. Innovationen führen dazu, dass analoge Produkte durch digitale ersetzt werden, die aber aus Nutzersicht den gleichen Zweck erfüllen (natürlich gibt es oft auch neue Funktionen bzw. Sachverhalte, die keine analogen Analoga haben).

\section{Perspektive eines oder mehrerer Beteiligter}

Der Gedanke der funktionalen Ersetzung blickt häufig nur aus Perspektive eines oder weniger Beteiligten/r auf den jeweiligen Sachverhalt. Darin liegt auch das Problem beim Analogieschluss: Dass ein bestimmtes digitales Produkt aus Nutzersicht ein analoges Produkt ersetzt (die gleiche Funktion hat), bedeutet nicht, dass es auch für andere Betroffene oder die Allgemeinheit funktional gleich ist (d.h. in gleicher Weise deren Interessen beeinträchtigt oder fördert). Nur dann aber wäre die Analogie gerechtfertigt. Eine eindimensionale Betrachtung digitaler Innovationsprozesse birgt die Gefahr, die tatsächliche Komplexität des Sachverhalts und die mit ihr einhergehenden normativen Implikationen nur unzureichend einzufangen. Als Konsequenz können sich die oben aufgezeigten Risiken eines unvollständigen Vergleichs verwirklichen. 


\section{Beispiel: digitale und analoge Medien}

Ein Beispiel der funktionalen Ersetzung ist der Bereich der Unterhaltungsmedien. Analoge Medien (Buch, Schallplatte, Videokassette) werden durch digitale körperliche Medien (CD, DVD) und schließlich durch unkörperliche digitale Medien (Download von Inhalten und schließlich Streaming von Inhalten) abgelöst. ${ }^{14}$ An der Entscheidung UsedSoft wird deutlich, dass die Sicht eines oder mehrerer Beteiligten/r, auch wenn es um deren wirtschaftliche Interessen geht, gerade nicht mit der umfassenderen volkswirtschaftlichen bzw. wohlfahrtsökonomischen Sicht gleichgesetzt werden kann. ${ }^{15}$ Der EuGH hielt den Download von Software mit dem Erwerb als Datenträger für "wirtschaftlich gesehen vergleichbar“. ${ }^{16}$ Dies ist allenfalls für die wirtschaftliche Bedeutung aus Erwerbersicht zutreffend, jedoch nicht für die volkswirtschaftliche Bedeutung, die für die rechtliche Würdigung hier (es ging um die Wirkung von Immaterialgüterrechten) relevant ist. ${ }^{17}$

\section{F. Intuitive Vergleiche}

\section{Intuition als eingeschränkter Blickwinkel}

Ein weiterer Fall regelmäßig unvollständiger Vergleiche sind solche, die auf bloßer Intuition beruhen, d.h. bei einer Intuition stehenbleiben und diese nicht mehr weiter hinterfragen. Analoges und Digitales werden dabei verglichen, weil sich ein solcher Vergleich aufgrund des Sprachgebrauchs oder anderer Umstände „aufdrängt“. In der Regel wird auch hier nur auf einen oder wenige Aspekte geblickt (wie beim Ersetzungsgedanken), sodass auch nur die Interessen bestimmter Beteiligter berücksichtigt werden und die Komplexität der normativen Gemengelage nur unzureichend zum Ausdruck kommt.

14 Zech, ZGE 2013, 368.

15 EuGH, Urt. v. 3.7.2012 - C-128/11 = ECLI:EU:C:2012:407 (UsedSoft GmbH/ Oracle International Corp.); zu E-Books jetzt anders EuGH, Urt. v. 19.12.2019 C-263/18 = ECLI:EU:C:2019:1111 (Nederlands Uitgeversverbond u.a./Tom Kabinet Internet BV u.a.).

16 EuGH, Urt. v. 3.7.2012 - C-128/11 = ECLI:EU:C:2012:407 (UsedSoft GmbH/ Oracle International Corp.), Rn. 61.

17 Zur volkswirtschaftlichen Einordnung der UsedSoft-Entscheidung Kerber, ZGE 2016, 149. Vgl. auch Perzanowski/Schultz, The End of Ownership, 2016, S. 184. 


\section{Beispiel: Daten als Rohstoff}

Ein wichtiges Beispiel für einen intuitiven Vergleich ist derjenige von Daten und Öl. Zwar kann dieser auch der bloßen Veranschaulichung dienen, es besteht aber die Gefahr, dass der Vergleich auch normativ aufgeladen wird, ohne dass ein echter Interessenvergleich stattfindet. So etwa bei der Frage der Eigentumsfähigkeit: Beide, Daten und Öl, so die Grundidee, werden gehandelt, beide dienen als Wirtschaftsgut. Dieser intuitive Vergleich lässt aber wichtige ökonomische und damit rechtlich relevante Unterschiede außer Acht.

Hier zeigt sich der Mehrwert einer ökonomischen Betrachtung. Aus ökonomischer Perspektive ist ein ganz entscheidendes Merkmal von Daten, dass sie - wie viele andere unkörperliche Güter auch - in ihrer Nutzung nicht-rival und nicht-exklusiv sind. Aus der Möglichkeit ihrer Vervielfältigung ergibt sich, dass Daten von beliebig vielen Akteuren genutzt werden können, ohne dass die Nutzung des einen der gleichzeitigen Nutzung des anderen entgegensteht (fehlende Rivalität). Und Daten, die einmal öffentlich zugänglich sind, lassen sich nicht mehr exklusiv nutzen, wobei technische Schutzmaßnahmen hier Abhilfe schaffen können (eingeschränkte Exklusivität). ${ }^{18}$

Rechtliche Regeln, die an diese ökonomischen Eigenschaften bzw. Kategorien anknüpfen, können daher nicht ohne weiteres analog angewendet werden. Sacheigentum, das der effizienten Zuweisung knapper Güter (Allokationseffizienz) dient, passt nicht. ${ }^{19}$ Das Gleiche gilt für den Besitzschutz, der an die faktische Exklusivität des Besitzes anknüpft. ${ }^{20}$ Aber auch immaterialgüterrechtliche Regelungen, denen vor allem ein Anreizgedanke betreffend die Gütererzeugung (Produktionseffizienz) zugrunde liegt, passen nicht ohne weiteres, wenn die Erzeugung von Rohdaten mit immer geringeren Kosten möglich ist. Es bestünde dann die Gefahr, dass ihre Anwendung - ebenso wie eine Anwendung von Sacheigentumsregeln - zu einer unnötigen bzw. ökonomisch nicht sinnvollen Verknappung von Daten führt. Eine analoge Anwendung sachenrechtlicher oder immaterialgüterrechtlicher Regelungen würde, wie die Einnahme der ökonomischen

18 Geheimhaltung bedeutet faktische Exklusivität, durch Offenbarung wird das Geheimnis zerstört. Exklusivität kann auch rechtlich durch Ausschließlichkeitsrechte vermittelt werden.

19 Specht, CR 2016, 288 (291f.); Steinrötter, MMR 2017, 731 (732); Zech, GRUR 2015, 1151 (1159); a.A. Hoeren, MMR 2013, 486.

20 Zech, in: Pertot (Hrsg.), Rechte an Daten, 2020, S. 91, 94 ff. 
Perspektive zeigt, daher auf einem unvollständigen Vergleich beruhen, der Interessen der Allgemeinheit außer Acht lässt.

Zusammenfassend lässt sich also festhalten: Daten und ganz allgemein viele unkörperliche Güter zeigen ökonomische Besonderheiten, die sie von Sachen unterscheiden. Daher verbietet sich eine intuitive Gleichstellung von körperlichen und unkörperlichen Wirtschaftsgütern.

\section{Beispiel: künstliche Intelligenz}

Ein weiteres Beispiel für einen intuitiven Vergleich, der zu nicht gerechtfertigten Analogieschlüssen (ver)führen kann, ist derjenige von künstlicher und natürlicher Intelligenz, d.h. von digitalen Systemen und Menschen. Wie bereits ausgeführt ist der Begriff der künstlichen Intelligenz ohnehin nicht sehr trennscharf und lässt sich am besten als der Versuch definieren, Leistungen natürlicher Intelligenz mit technischen Mitteln zu vollbringen (technische statt "natürlicher" Informationsverarbeitung).

Diskutiert wird, ob Regelungen, die auf den Einsatz von Hilfspersonen zugeschnitten sind, auch auf digitale Systeme angewendet werden können, etwa in der deliktischen oder vertraglichen Haftung, ${ }^{21}$ beim Vertragsschluss bzw. beim Tätigen von Rechtsgeschäften, ${ }^{22}$ oder bei der Schaffung unkörperlicher Güter (Erfinder im Patentrecht, Werkschöpfer im Urheberrecht etc.). Es geht um die Zurechnung bestimmter Erfolge. ${ }^{23}$

Hier zeigen sich Unterschiede zwischen natürlichen und künstlichen Intelligenzen bzw. Menschen und Maschinen, die einen intuitiven Vergleich verbieten, etwa in der körperlichen Verfasstheit (und damit Erkennbarkeit für Dritte) oder dem Innehaben eigenen Vermögens (und damit einer Haftungsmasse). Das bedeutet nicht, dass Analogien zwischen Menschen (bzw. Hilfspersonen) und Maschinen bzw. die analoge Anwendung entsprechender rechtlicher Regelungen auf digitale Systeme in jedem Fall unterbleiben müssen. Sie setzen aber einen Interessenvergleich voraus, der sämtliche relevanten Aspekte in den Blick nimmt, insbesondere auch die Auswirkungen für Betroffene, die nicht als Entwickler, Hersteller, Betreiber oder Benutzer künstlicher Intelligenz beteiligt sind, und für die Allgemeinheit.

21 Dazu z.B. Teubner, AcP 218 (2018), 155 (185 ff., 189 ff.); Zech, Gutachten A zum 73. DJT, S. $76 \mathrm{ff}$.

22 Dazu z.B. Schirmer, JZ 2016, 660 (664); Teubner, AcP 218 (2018), 155 (177 ff., 184).

23 Statt vieler Hacker, RW 2018, 243; Zech, Gutachten A zum 73. DJT, S. 76 ff. 
Eine vollständige Interessenanalyse muss auch Unterschiede zwischen dem Einsatz von Hilfspersonen und dem Einsatz von Informationstechnologie in den Blick nehmen: neben der Publizität/Erkennbarkeit und Haftungsmasse auch die Kontrolle des Einsetzenden bzw. Autonomie des Eingesetzten und die Vermeidbarkeit für Betroffene. Geht es etwa um Haftungsfragen, so ist der Aspekt der Risikozuweisung das entscheidende Vergleichsmerkmal, geht es um das Immaterialgüterrecht, die Zuweisung sozialer Vorteile neuer Immaterialgüter.

Nur soweit sich eine sachgerechte Zurechnung der Erfolge bzw. Effekte des Einsatzes digitaler Systeme nicht durch Anwendung von Regelungen bewerkstelligen lässt, die auf dem Werkzeugcharakter digitaler Systeme fußen, sollte über eine analoge Anwendung rechtlicher Regelungen zu Hilfspersonen nachgedacht werden.

Zusammenfassend lässt sich also warnen: Keine intuitive Gleichstellung von natürlicher und künstlicher Intelligenz, technischer und rechtlicher Autonomie. Eine Analogie aufgrund umfassender Interessenabwägung wird dadurch aber nicht ausgeschlossen.

\section{G. Schlussfolgerungen}

Fragt man abschließend nach Potential und Grenzen der Analogie zum Analogen, so lässt sich wie zu erwarten eine differenzierende Antwort geben: Sie birgt Nutzen und Gefahren - den Nutzen vollständiger Vergleiche, die Gefahr unvollständiger Vergleiche, insbesondere durch Ersetzungsgedanken und bloße Intuition.

Als Ausblick soll darauf hingewiesen werden, dass sich aus dem Vergleich von digitalen und analogen Sachverhalten nicht nur Analogieschlüsse, sondern auch teleologische Reduktionen rechtfertigen lassen. Normen, die für analoge Sachverhalte geschaffen wurden und nach deren Digitalisierung zu funktionswidrigen Ergebnissen führen, können entsprechend teleologisch reduziert werden. ${ }^{24}$

Rechtliche Analogieschlüsse sind ein methodischer Weg, mit sich schnell ändernden Lebenssachverhalten umzugehen. Die Analogie als Instrument der Rechtsfortbildung ist aus rechtsstaatlicher und demokrati-

24 So etwa (wenn auch im Wege der einfachen Auslegung) \$903 Satz 1 BGB, der nicht das Kopieren von Datenträgern umfasst, s. Zech, AcP 219 (2019), 488 (585); anders Raue, NJW 2019, 2425 (2426 ff.), der ein Recht am Datenzugriff an der eigenen Sache befürwortet. 
scher Perspektive nicht unproblematisch. Sie ermöglicht es der Judikative, dem Rechtsgewährungsgebot zu genügen, darf aber die Legislative nicht aus der Verantwortung entlassen. Daher wird es zur Schließung rechtlicher Lücken, die die Digitalisierung reißt, oftmals eines Gesetzes und damit eines demokratisch legitimierten Aktes bedürfen. 